NIST Technical Note 2080

\title{
On the Cooling of a $360^{\circ}$ Video Camera to Observe Fire Dynamics \\ In Situ
}

Jiann C. Yang

This publication is available free of charge from:

https://doi.org/10.6028/NIST.TN.2080

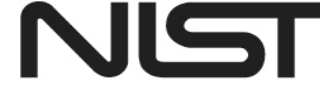

National Institute of Standards and Technology U.S. Department of Commerce 
NIST Technical Note 2080

\section{On the Cooling of a $360^{\circ}$ Video Camera to Observe Fire Dynamics In Situ}

This publication is available free of charge from:

https://doi.org/10.6028/NIST.TN.2080

Fire Research Division

Engineering Laboratory

December 2019

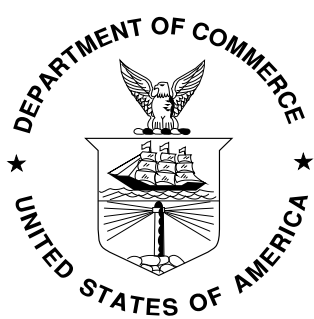

U.S. Department of Commerce

Wilbur L. Ross, Jr., Secretary

National Institute of Standards and Technology Walter Copan, NIST Director and Undersecretary of Commerce for Standards and Technology 
Certain commercial entities, equipment, or materials may be identified in this document in order to describe an experimental procedure or concept adequately. Such identification is not intended to imply recommendation or endorsement by the National Institute of Standards and Technology, nor is it intended to imply that the entities, materials, or equipment are necessarily the best available for the purpose.

National Institute of Standards and Technology Technical Note 2080 Natl. Inst. Stand. Technol. Tech. Note 2080, 15 pages (December 2019) CODEN: NTNOEF

This publication is available free of charge from: https://doi.org/10.6028/NIST.TN.2080 


\begin{abstract}
An analysis was performed to provide design guidance on the cooling of an NIST-developed in situ fire visualization device, called Burn Observation Bubble (BOB). The analysis was to address the question of how long the device can operate in a fire without being damaged by the fire. The analysis included consideration of single-pass and recirculating cooling systems. The resulting design equations depend on the fire scenario, the cooling water flow rate, and the volume ratio of the device volume to recirculating cooling water reservoir. In the limit of an infinite recirculating cooling water reservoir, the design equations reduce to those of a single-pass system. Sample calculations were given to illustrate the applicability of the equations.
\end{abstract}

\title{
Key words
}

Cooling; fire visualization; modelling. 


\section{Table of Contents}

1. Introduction ......................................................................................................................... 1

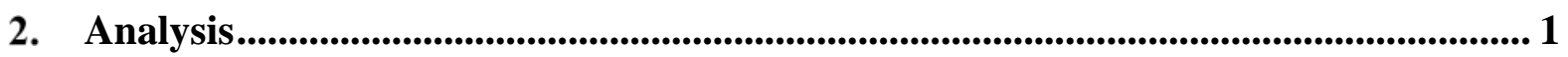

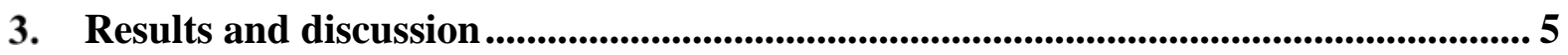

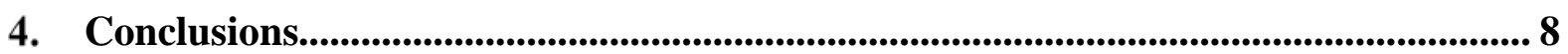

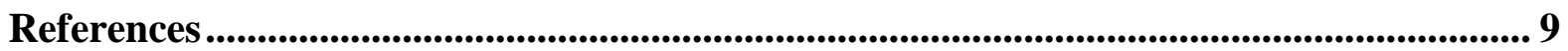

\section{List of Figures}

Fig. 1. Thermodynamic model representation of cooling of the spherical enclosure using a recirculating cooling water reservoir.

Fig. 2. Calculated temporal variations of $\tilde{T}_{1}$ and $\tilde{T}_{2}$ at $\tilde{Q}_{1}=3.11 \times 10^{-2}$ and different $\tau(=0.03,0.015,0)$. Solid lines represent $\tilde{T}_{1}$ (with $\tau=0.03$ in red, $\tau=0.015$ in black, and $\tau=0$ in blue), and dash lines represent $\tilde{T}_{2}$ (with $\tau=0.03$ in red, $\tau=0.015$ in black, and $\tau=0$ in blue).

Fig. 3. Calculated temporal variations of $\tilde{T}_{1}$ and $\tilde{T}_{2}$ at $\tau=0.03$ and different $\tilde{Q}_{1}\left(=3.11 \times 10^{-2}, 4.67 \times 10^{-2}, 9.33 \times 10^{-2}\right.$ ). Solid lines represent $\tilde{T}_{1}$ (with $\tilde{Q}_{1}=3.11 \times 10^{-2}$ in red, $\tilde{Q}_{1}=4.67 \times 10^{-2}$ in black, and $\tilde{Q}_{1}=9.33 \times 10^{-2}$ in blue), and dash lines represent $\tilde{T}_{2}$ (with $\tilde{Q}_{1}=3.11 \times 10^{-2}$ in red, $\tilde{Q}_{1}=4.67 \times 10^{-2}$ in black, and $\tilde{Q}_{1}=9.33 \times 10^{-2}$ in blue)..................... 8 


\section{Glossary}

$c_{p} \quad$ specific heat of water $(\mathrm{J} / \mathrm{kg}-\mathrm{K})$

$\dot{Q}_{1} \quad$ net heat flow rate to the spherical enclosure (W)

$\tilde{Q}_{1} \quad$ dimensionless $\dot{Q}_{1}(--)$

$\dot{Q}_{2} \quad$ net heat loss rate from the cooling water reservoir (W)

$\tilde{Q}_{2} \quad$ dimensionless $\dot{Q}_{2}(--)$

$t$ time (s)

$\tilde{t} \quad$ dimensionless time (--)

$T_{1} \quad$ temperature of the spherical enclosure (K)

$\tilde{T}_{1} \quad$ dimensionless temperature of the spherical enclosure (--)

$T_{2} \quad$ temperature of the cooling water reservoir (K)

$\tilde{T}_{2}$ dimensionless temperature of the cooling water reservoir (--)

$T_{\infty} \quad$ ambient room temperature (K)

$\dot{V} \quad$ volumetric flow rate $\left(\mathrm{m}^{3} / \mathrm{s}\right)$

$V_{1} \quad$ volume of the spherical enclosure $\left(\mathrm{m}^{3}\right)$

$V_{2} \quad$ volume of the cooling water reservoir $\left(\mathrm{m}^{3}\right)$

Greek symbols

$\rho \quad$ density of water $\left(\mathrm{kg} / \mathrm{m}^{3}\right)$

$\tau \quad$ volume ratio of the spherical enclosure to the cooling water reservoir (--)

$\tau_{1} \quad$ residence time of the spherical enclosure (s)

$\tau_{2} \quad$ residence time of the cooling water reservoir (s) 


\section{Introduction}

The Burn Observation Bubble (known as its acronym BOB) is a device which consists of a commercially available off-the-shelf digital $360^{\circ}$ view waterproof camera inside a transparent glass sphere filled with flowing water. The device, which was conceived, designed, and constructed by Hoehler [1] of NIST, is intended to be used to observe fire dynamics behavior inside a fire in laboratories and fields. The functions of the water are to cool the camera and to absorb infrared radiation from the intense fire, reducing camera pixel saturation for better video quality. In laboratories where there is ample water supply, the spherical enclosure is normally cooled by flowing water in a single-pass configuration. For field operations (e.g., observing a wildland fire) where water supply is limited, the enclosure is normally cooled using a recirculating water system with a large water reservoir buried underground to shield the reservoir from exposure to the intense fire. Since it is a closed loop system with heat transfer into the system greater than heat loss to the surroundings, the recirculating water will get warmer and warmer as the device is continuously exposed to a fire. The temporal temperature variations of the spherical enclosure and the cooling water reservoir will dictate how long the video camera can operate in that thermal environment before it is damaged or destroyed by the fire. For a given fire scenario where a given spherical enclosure configuration is to be used, the important design parameters for the cooling water system are the size of the cooling water reservoir, the water flow rate, and the time to reach the internal spherical enclosure temperature that could thermally damage the video camera and render it inoperable. In this technical note, an analysis is given to provide design guidance for the cooling system, recirculating or single-pass, to be used with the spherical enclosure.

\section{Analysis}

Figure 1 is a thermodynamic model used to perform the analysis. For the spherical enclosure (System 1) and assuming the system is well-mixed and the video camera inside the spherical enclosure is in thermal equilibrium with the water immersing the camera (i.e., the temperature of the video camera is equal to $T_{1}$ ), an energy balance results in the following equation.

$$
\rho c_{p} V_{1} \frac{\mathrm{d} T_{1}}{\mathrm{~d} t}=\rho \dot{V} c_{p}\left(T_{2}-T_{1}\right)+\dot{Q}_{1}
$$

Similarly, for the cooling water reservoir (System 2),

$$
\rho c_{p} V_{2} \frac{\mathrm{d} T_{2}}{\mathrm{~d} t}=\rho \dot{V} c_{p}\left(T_{1}-T_{2}\right)-\dot{Q}_{2}
$$

In the following analysis, heat transfer in the piping system is assumed to be negligible. Define the following dimensionless parameters:

$$
\tau_{1} \equiv \frac{V_{1}}{\dot{V}} \quad \tau_{2} \equiv \frac{V_{2}}{\dot{V}} \quad \tau \equiv \frac{\tau_{1}}{\tau_{2}}=\frac{V_{1}}{V_{2}}
$$




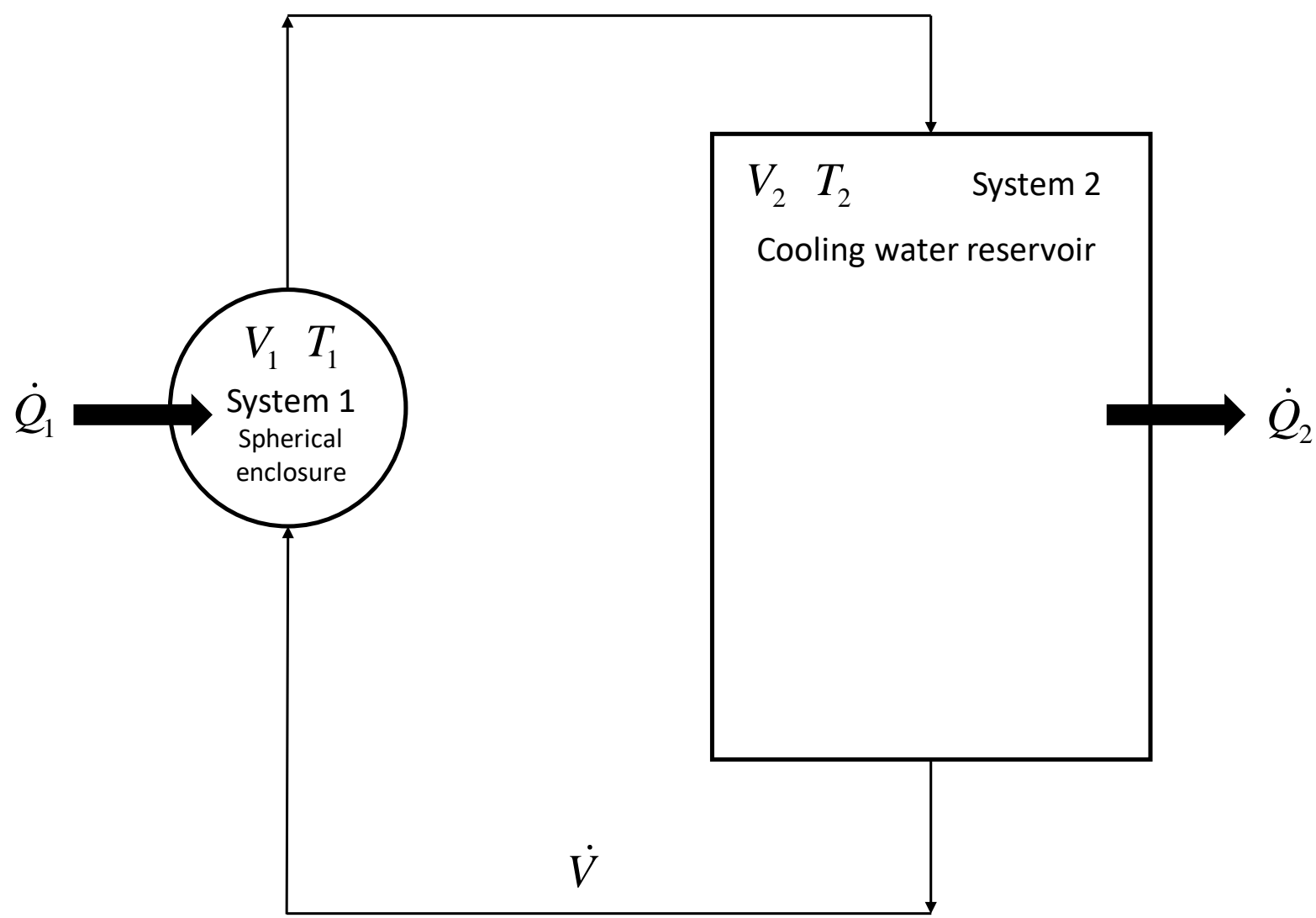

Fig. 1. Thermodynamic model representation of cooling of the spherical enclosure using a recirculating cooling water reservoir.

$\tilde{t} \equiv \frac{t}{\tau_{1}}$

$\tilde{T}_{1} \equiv \frac{T_{1}}{T_{\infty}} \quad \tilde{T}_{2} \equiv \frac{T_{2}}{T_{\infty}}$

$\tilde{Q}_{1} \equiv \frac{\dot{Q}_{1}}{\rho c_{p} T_{\infty} \dot{V}} \quad \tilde{Q}_{2} \equiv \frac{\dot{Q}_{2}}{\rho c_{p} T_{\infty} \dot{V}}$

Equations (1) and (2) then become

$$
\begin{gathered}
\frac{\mathrm{d} \tilde{T}_{1}}{\mathrm{~d} \tilde{t}}=\left(\tilde{T}_{2}-\tilde{T}_{1}\right)+\tilde{Q}_{1} \\
\frac{\mathrm{d} \tilde{T}_{2}}{\mathrm{~d} \tilde{t}}=\tau\left(\tilde{T}_{1}-\tilde{T}_{2}\right)-\tau \tilde{Q}_{2}
\end{gathered}
$$


Equations (3) and (4) can be solved using Laplace transform with the initial conditions $\tilde{T}_{1}(0)=\tilde{T}_{2}(0)=1$.

Taking the Laplace transform of Eq. (3),

$$
\mathcal{L}\left\{\frac{\mathrm{d} \tilde{T}_{1}}{\mathrm{~d} \tilde{t}}\right\}=\mathcal{L}\left\{\left(\tilde{T}_{2}-\tilde{T}_{1}\right)\right\}+\mathcal{L}\left\{\tilde{Q}_{1}\right\}
$$

with $\mathcal{L}\left\{\tilde{T}_{1}\right\} \equiv t_{1}(s)$ and $\mathcal{L}\left\{\tilde{T}_{2}\right\} \equiv t_{2}(s)$,

$$
(s+1) t_{1}-t_{2}=\frac{\tilde{Q}_{1}}{s}+1
$$

Taking the Laplace transform of Eq. (4),

$$
\begin{gathered}
\mathcal{L}\left\{\frac{\mathrm{d} \tilde{T}_{2}}{\mathrm{~d} \tilde{t}}\right\}=\mathcal{L}\left\{\tau\left(\tilde{T}_{1}-\tilde{T}_{2}\right)\right\}-\mathcal{L}\left\{\tau \tilde{Q}_{2}\right\} \\
-\tau t_{1}+(s+\tau) t_{2}=1-\frac{\tau \tilde{Q}_{2}}{s}
\end{gathered}
$$

Writing Eqs. (5) and (6) in matrix form,

$$
\left[\begin{array}{cc}
(s+1) & -1 \\
-\tau & (s+\tau)
\end{array}\right]\left[\begin{array}{l}
t_{1} \\
t_{2}
\end{array}\right]=\left[\begin{array}{c}
\frac{\tilde{Q}_{1}}{s}+1 \\
1-\frac{\tau \tilde{Q}_{2}}{s}
\end{array}\right]
$$

Solving for $t_{1}$ and $t_{2}$,

$$
\left[\begin{array}{l}
t_{1} \\
t_{2}
\end{array}\right]=\left[\begin{array}{cc}
(s+1) & -1 \\
-\tau & (s+\tau)
\end{array}\right]^{-1}\left[\begin{array}{c}
\frac{\tilde{Q}_{1}}{s}+1 \\
1-\frac{\tau \tilde{Q}_{2}}{s}
\end{array}\right]
$$

Evaluating the above inverse matrix, one obtains 


$$
\left[\begin{array}{l}
t_{1} \\
t_{2}
\end{array}\right]=\frac{1}{s^{2}+s+\tau s}\left[\begin{array}{cc}
(s+\tau) & 1 \\
\tau & (s+1)
\end{array}\right]\left[\begin{array}{c}
\frac{\tilde{Q}_{1}}{s}+1 \\
1-\frac{\tau \tilde{Q}_{2}}{s}
\end{array}\right]
$$

or

$$
\begin{gathered}
t_{1}=\frac{\tilde{Q}_{1}+s+\frac{\tau \tilde{Q}_{1}}{s}+\tau+1-\frac{\tau \tilde{Q}_{2}}{s}}{s^{2}+s+\tau s}=\frac{\tau\left(\tilde{Q}_{1}-\tilde{Q}_{2}\right)}{s\left(s^{2}+s+\tau s\right)}+\frac{s+\left(\tilde{Q}_{1}+\tau+1\right)}{s^{2}+s+\tau s} \\
t_{2}=\frac{\frac{\tau \tilde{Q}_{1}}{s}+\tau+s-\tau \tilde{Q}_{2}+1-\frac{\tau \tilde{Q}_{2}}{s}}{s^{2}+s+\tau s}=\frac{\tau\left(\tilde{Q}_{1}-\tilde{Q}_{2}\right)}{s\left(s^{2}+s+\tau s\right)}+\frac{s+\left(-\tau \tilde{Q}_{2}+\tau+1\right)}{s^{2}+s+\tau s}
\end{gathered}
$$

Taking the inverse Laplace transform of Eq. (10),

$$
\mathcal{L}^{-1}\left\{t_{1}\right\}=\tau\left(\tilde{Q}_{1}-\tilde{Q}_{2}\right) \mathcal{L}^{-1}\left\{\frac{1}{s\left(s^{2}+s+\tau s\right)}\right\}+\mathcal{L}^{-1}\left\{\frac{s+\left(\tilde{Q}_{1}+\tau+1\right)}{s^{2}+s+\tau s}\right\}
$$

with $\mathcal{L}^{-1}\left\{t_{1}\right\} \equiv \tilde{T}_{1}$ and evaluating the inverse transform, one obtains the dimensionless temporal variation of the spherical enclosure temperature in closed form.

$$
\begin{gathered}
\tilde{T}_{1}=\tau\left(\tilde{Q}_{1}-\tilde{Q}_{2}\right)\left\{\frac{\tilde{t}(\tau+1)+\exp [-(\tau+1) \tilde{t}]-1}{(\tau+1)^{2}}\right\}+\exp [-(\tau+1) \tilde{t}] \\
+\left(\tilde{Q}_{1}+\tau+1\right)\left\{\frac{1-\exp [-(\tau+1) \tilde{t}]}{(\tau+1)}\right\}
\end{gathered}
$$

Similarly, taking the inverse Laplace transform of Eq. (11),

$$
\mathcal{L}^{-1}\left\{t_{2}\right\}=\mathcal{L}^{-1}\left\{\frac{\tau\left(\tilde{Q}_{1}-\tilde{Q}_{2}\right)}{s\left(s^{2}+s+\tau s\right)}\right\}+\mathcal{L}^{-1}\left\{\frac{s+\left(-\tau \tilde{Q}_{2}+\tau+1\right)}{s^{2}+s+\tau s}\right\}
$$

with $\mathcal{L}^{-1}\left\{t_{2}\right\} \equiv \tilde{T}_{2}$ and evaluating the inverse transform, one obtains the dimensionless temporal variation of temperature in the cooling water reservoir in closed form. 


$$
\begin{gathered}
\tilde{T}_{2}=\tau\left(\tilde{Q}_{1}-\tilde{Q}_{2}\right)\left\{\frac{\tilde{t}(\tau+1)+\exp [-(\tau+1) \tilde{t}]-1}{(\tau+1)^{2}}\right\}+\exp [-(\tau+1) \tilde{t}] \\
+\left(-\tau \tilde{Q}_{2}+\tau+1\right)\left\{\frac{1-\exp [-(\tau+1) \tilde{t}]}{(\tau+1)}\right\}
\end{gathered}
$$

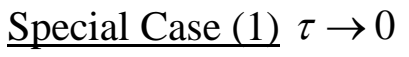

When the cooling water reservoir is infinite, $\tau \rightarrow 0$. Then Eqs. (12) and (13) become

$$
\begin{gathered}
\lim _{\tau \rightarrow 0} \tilde{T}_{1}=1+\tilde{Q}_{1}[1-\exp (-\tilde{t})] \\
\lim _{\tau \rightarrow 0} \tilde{T}_{2}=1
\end{gathered}
$$

Special Case (2) Single-pass cooling

If the spherical enclosure is operated in a single-pass mode without the cooling water reservoir, the temperature rise in the spherical enclosure can be simply calculated from Eq. (3) with $\tilde{T}_{2}=1$.

$$
\frac{\mathrm{d} \tilde{T}_{1}}{\mathrm{~d} \tilde{t}}=\left(1-\tilde{T}_{1}\right)+\tilde{Q}_{1}
$$

Integrating Eq. (16), one obtains

$$
\tilde{T}_{1}=1+\tilde{Q}_{1}[1-\exp (-\tilde{t})]
$$

Equation (17) is identical to Eq. (14), which is expected because having a cooling water reservoir with infinite volume in a recirculating loop is equivalent to having a single-pass cooling system.

At $\tilde{t} \rightarrow \infty$, Eq. (17) becomes

$$
\lim _{\tilde{t} \rightarrow \infty} \tilde{T}_{1}=1+\tilde{Q}_{1}
$$

\section{Results and discussion}

Equations (12) and (13) show that $\tilde{T}_{1}$ and $\tilde{T}_{2}$ depend on three dimensionless parameters, $\tau$, $\tilde{Q}_{1}$, and $\tilde{Q}_{2}$. The intended operating dimensionless temperature of the video camera typically 
is $\tilde{T}_{1} \leq 1.05$. If one assumes $\tilde{Q}_{2}=0$ (i.e., the cooling water reservoir is adiabatic, which can be considered as a conservative condition) and for a given $\tilde{Q}_{1}$ (implying a known fire scenario $\dot{Q}_{1}$ and a known water flow rate $\dot{V}$ ) and an intended dimensionless period to surround the spherical enclosure in a fire, one could size the cooling water reservoir by varying $\tau$ using Eq. (12). To prevent thermal damage to the video camera, the intended dimensionless operating period should be less than or equal to the dimensionless time $\tilde{t}$ when $\tilde{T}_{1}=1.05$. Conversely, for a given $\tau$ (fixed cooling water reservoir), one could determine under what fire scenario $\dot{Q}_{1}$ for a given water flow rate $\dot{V}$ (or what water flow rate $\dot{V}$ for a given fire scenario $\dot{Q}_{1}$ ) how long the video camera can operate by varying $\tilde{Q}_{1}$ using Eq. (12) before the spherical enclosure exceeds the intended operating dimensionless temperature $\left(\tilde{T}_{1}=1.05\right)$ of the video camera.

Figures 2 and 3 are calculated examples using the following parameters: $\rho=1000 \mathrm{~kg} / \mathrm{m}^{3}$, $c_{p}=4200 \mathrm{~J} / \mathrm{kg}-\mathrm{K}, T_{\infty}=295 \mathrm{~K}$, and $\tilde{Q}_{2}=0$. The radius of the spherical enclosure was fixed at $0.0762 \mathrm{~m}$ (3 in). For the fire scenario, a value of $\dot{Q}_{1}=7297 \mathrm{~W}$ was used by assuming that a constant heat flux of $100 \mathrm{~kW} / \mathrm{m}^{2}$ was imposed on the spherical enclosure.

Figure 2 shows the effect of $\tau$ on heating up the spherical enclosure for given fire scenario and flow rate (constant $\tilde{Q}_{1}$ ). The results illustrated in Figure 2 were obtained using a fixed value of $\tilde{Q}_{1}=3.11 \times 10^{-2}$, which was based on $\dot{Q}_{1}=7297 \mathrm{~W}$ and $\dot{V}=18.93 \times 10^{-5} \mathrm{~m}^{3} / \mathrm{s}$ (3 gal $/ \mathrm{min}$ ), and different $\tau$ values. For a given spherical enclosure size $\left(V_{1}=\right.$ constant $)$, varying $\tau$ amounts to sizing $V_{2}$ (the cooling water reservoir volume).

Figure 3 shows the effect of $\tilde{Q}_{1}$ on heating up the spherical enclosure for given fire scenario (constant $\dot{Q}_{1}$ ) and cooling water reservoir size (constant $\tau$ ). Figure 3 represents the results calculated using fixed $\tau(=0.03)$ and different $\tilde{Q}_{1}\left(=3.11 \times 10^{-2}, 4.67 \times 10^{-2}, 9.33 \times 10^{-2}\right)$. These $\tilde{Q}_{1}$ values were obtained using $\dot{V}=18.93 \times 10^{-5} \mathrm{~m}^{3} / \mathrm{s}$ ( $\left.3 \mathrm{gal} / \mathrm{min}\right), \dot{V}=12.62 \times 10^{-5} \mathrm{~m}^{3} / \mathrm{s}$ (2 gal $/ \mathrm{min}$ ), and $\dot{V}=6.31 \times 10^{-5} \mathrm{~m}^{3} / \mathrm{s}(1 \mathrm{gal} / \mathrm{min})$ respectively. Note that Figure 3 also illustrates the effect of $\dot{Q}_{1}$ on heating up the spherical enclosure for constant $\dot{V}$ and constant $\tau$ since same $\tilde{Q}_{1}$ can be obtained by varying either $\dot{Q}_{1}$ or $\dot{V}$. In this case, the results will determine how long a given spherical enclosure (known $\tau, \dot{V}$ ) can be safely used to protect the video camera from thermal damage in a fire scenario specified by $\dot{Q}_{1}$. 


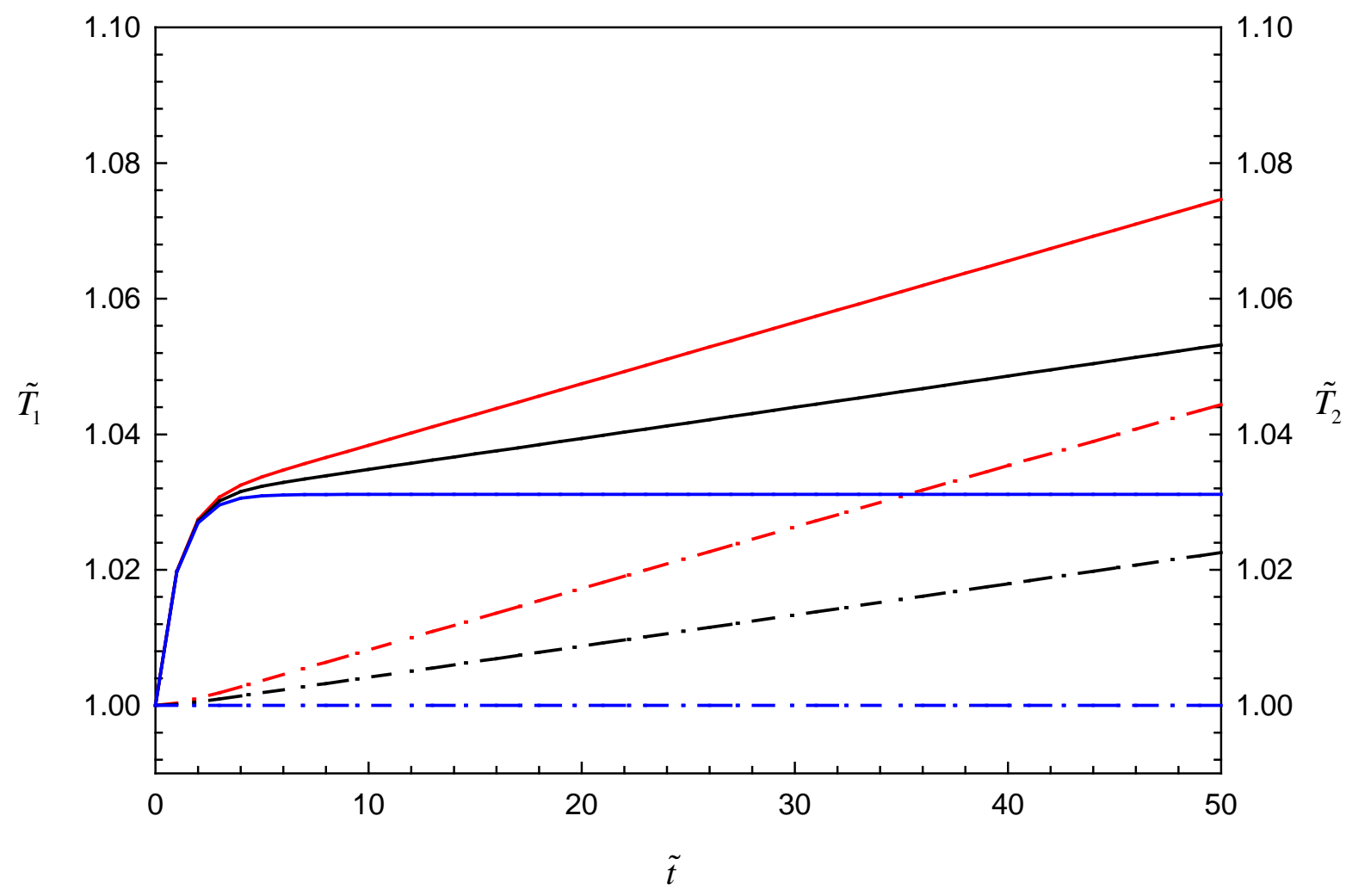

Fig. 2. Calculated temporal variations of $\tilde{T}_{1}$ and $\tilde{T}_{2}$ at $\tilde{Q}_{1}=3.11 \times 10^{-2}$ and different $\tau(=0.03,0.015,0)$. Solid lines represent $\tilde{T}_{1}$ (with $\tau=0.03$ in red, $\tau=0.015$ in black, and $\tau=0$ in blue), and dash lines represent $\tilde{T}_{2}$ (with $\tau=0.03$ in red, $\tau=0.015$ in black, and $\tau=0$ in blue). 


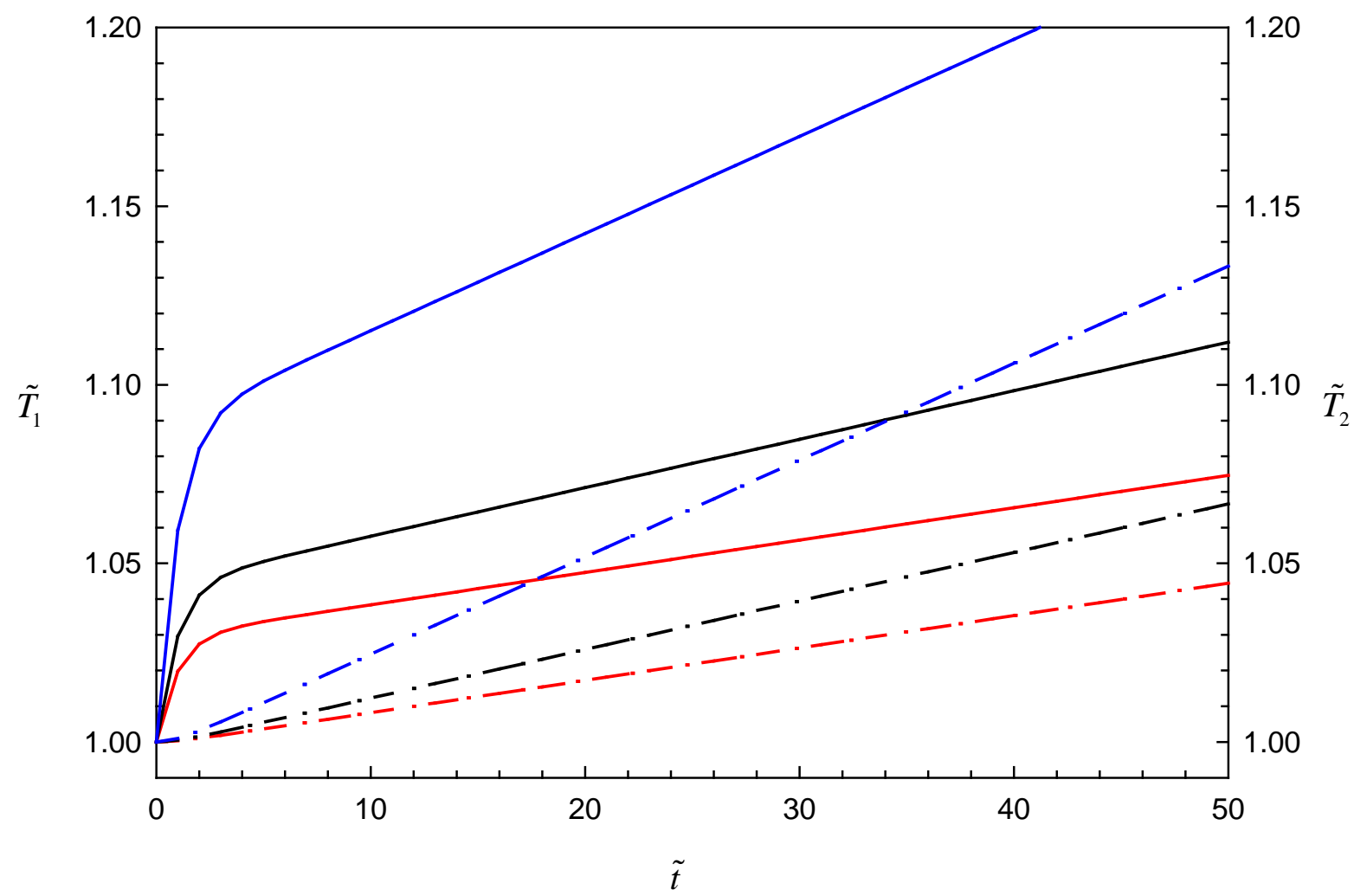

Fig. 3. Calculated temporal variations of $\tilde{T}_{1}$ and $\tilde{T}_{2}$ at $\tau=0.03$ and different $\tilde{Q}_{1}\left(=3.11 \times 10^{-2}, 4.67 \times 10^{-2}, 9.33 \times 10^{-2}\right)$. Solid lines represent $\tilde{T}_{1}$ (with $\tilde{Q}_{1}=3.11 \times 10^{-2}$ in red, $\tilde{Q}_{1}=4.67 \times 10^{-2}$ in black, and $\tilde{Q}_{1}=9.33 \times 10^{-2}$ in blue), and dash lines represent $\tilde{T}_{2}$ (with $\tilde{Q}_{1}=3.11 \times 10^{-2}$ in red, $\tilde{Q}_{1}=4.67 \times 10^{-2}$ in black, and $\tilde{Q}_{1}=9.33 \times 10^{-2}$ in blue).

\section{Conclusions}

An analysis was performed to provide design calculations on the cooling water inside a spherical enclosure to thermally protect a $360^{\circ}$ view digital video camera immersed inside the enclosure during fire operation. The analysis was intended to address the question of how long the spherical enclosure can be water-cooled in a fire without the video camera being damaged by the fire. The analysis included the behavior for both single-pass and recirculating cooling systems. The resulting design equations depend only on the fire scenario, cooling water flow rate, and the volume ratio of the spherical enclosure to recirculating cooling water reservoir. In the limit of an infinite recirculating cooling water reservoir, the design equations reduce to those of a single-pass system. Sample calculations were given to illustrate the applicability of the equations. Due to the algebraic nature and the simplicity of the design equations, calculations could be easily performed using spreadsheet software. 


\section{References}

[1] Hoehler M (2020) On the Development of a Transparent Enclosure for $360^{\circ}$ Video Cameras to Observe Severe Fires In Situ. Fire Safety Science (accepted for publication). 